\title{
Preoperative prediction score of hepatocellular carcinoma recurrence in living donor liver transplantation: validation of SNAPP score developed at Asan Medical Center
}

\author{
Seok-Hwan Kim', Deok-Bog Moon ${ }^{2}$ \\ ${ }^{1}$ Division of Transplantation, Department of Surgery, Chungnam National University Hospital, Daejeon, Korea \\ ${ }^{2}$ Division of Transplantation, Department of Surgery, Asan Medical Center, University of Ulsan College of Medicine, Seoul, Korea
}

Background: The previously proposed scoring systems are not readily available due to the lack of simplicity for predicting hepatocellular carcinoma (HCC) recurrence. We aimed to develop and validate the new score system, which can predict HCC recurrence after living donor liver transplantation (LDLT) by using morphologic and biologic data.

Methods: Predictors for HCC recurrence after LDLT were developed $(n=627)$ and validated $(n=806)$ in 1,433 patients who could collect information to date between 2007 and 2016 at Asan Medical Center to create the Score for Neonatal Acute Physiology (SNAPP) score (tumor size and number, alpha-fetoprotein, vitamin $\mathrm{K}$ absence-II, and positron emission tomography).

Results: On logistic regression based on 3-year recurrence-free survival, the SNAPP factors were independently associated with HCC recurrence. The SNAPP score was highly predictive of HCC recurrence (C statistic, 0.920 ), and 5-year post-liver transplantation recurrence rates were significantly different between low, intermediate, and high SNAPP score groups. The performance of the SNAPP scores (C-index, $0.840 ; 95 \%$ confidence interval, 0.801-0.876) on predicting tumor recurrence after LDLT was better than that of the NYCA (New York/California), the RETREAT (Risk Estimation of Tumor Recurrence after Transplant), and the MoRAL (Model of Recurrence after Liver Transplant) scores.

Conclusions: The SNAPP score provides excellent prognostication after LDLT for HCC patients. Hence, we can help voluntary patients' decisions about whether to undergo LDLT or not.

Corresponding author: Deok-Bog Moon

E-mail:mdb1@amc.seoul.kr

\section{(c) The Korean Society for Transplantation}

This is an Open Access article distributed under the terms of the Creative Commons Attribution Non-Commercial License (http://creativecommons.org/licenses/by-nc/4.0/) which permits unrestricted non-commercial use, distribution, and reproduction in any medium, provided the original work is properly cited 